\title{
Tanimoto Gaussian Kernelized Feature Extraction Based Multinomial Gentleboost Machine Learning for Multi-Spectral Aerial Image Classification
}

\author{
A.Gokila Vani, V. Saravanan
}

\begin{abstract}
Aerial images provide a landscape view of earth surfaces that utilized to monitor the large areas. Each Aerial image comprises the different scenes to identify the objects on the digital maps. The several methodologies have been developed to solve the problem of the scene classification using input aerial images. The method does not improve the classification performance using more aerial images. In order to improve the classification performance, a Tanimoto Gaussian Kernelized Feature Extraction Based Multinomial GentleBoost Classification (TGKFE-MGBC) technique is introduced. The TGKFE-MGBC technique comprises three major processes namely object-based segmentation, feature extraction and aerial image scene classification. At first, object-based segmentation partitions the aerial image into several sub-bands. Aerial image with more than two objects is called as multi-spectral. The objects in spectral bands are identified by Tanimoto pixel similarity measure. This process helps to reduce the feature extraction time. Each object has different features like shape, size, color, texture and so on. After that, Gaussian Kernelized Feature Extraction is carried out to extracts the features from the objects with minimal time. Finally, the Multinomial GentleBoost Classification is applied for categorizing the scenes into different classes with the extracted features. The GentleBoost is an ensemble technique uses multinomial nä̈ve Bayes probabilistic classifier as a weak learner and it combines to makes a strong one for classifying the scenes. The strong classifier result improves the aerial image scene classification accuracy and minimizes the false positive rate. Simulation is conducted using aerial image database with different factors such as feature extraction time, aerial image scene classification accuracy and false positive rate. The results showed that the TGKFE-MGBC technique effectively improves the aerial image scene classification accuracy and minimizes the feature extraction time as well as the false positive rate.
\end{abstract}

Keywords : aerial image, object-based segmentation, Tanimoto pixel similarity, Gaussian Kernelized Feature Extraction, Multinomial GentleBoost scene Classification, weak learner, naïve Bayes probabilistic classifier .

Revised Manuscript Received on July 08, 2019

A.Gokila Vani, Ph.D. Scholar, Hindusthan College of Arts and Science,Coimbatore - 641028.

Dr. V. Saravanan, Associate Professor \& HEAD, Hindusthan College of Arts and Science, Department of IT, Hindusthan College of Arts and Science,Coimbatore - 641028

\section{INTRODUCTION}

Detection of small objects in aerial images has diverse applications in various domains, such as surveillance, military, remote sensing, and so on. But the accurate detection is a demanding issue, mainly due to the major interclass similarity between the objects in environments. Therefore, the scene classification is performed with the number of objects. Besides, the computational complexity issues arise during the scene classification. The feature extraction and segmentation process are carried out before the classification for minimizing this complexity.

A recurrent neural network (RNN) classifier was introduced in [1] to perform satellite image classification and discover the relevant features. However, the RNN does not improve classification accuracy since it does not minimize the training error. Block-based semantic classification technique was introduced in [2] with high quality multispectral aerial images. The technique only provides high classification accuracy when small training sets were used.

A two-stream deep fusion approach was introduced in [3] for categorizing the aerial scene with high-quality remote sensing images using ELM classifier with fused features. Though the approach provides better classification results, the false positive rate was not minimized.

An accurate scene classification with local and global spatial features was presented in [4]. The approach does not minimize the time complexity in the feature extraction. A Deep convolutional neural network (DCNNs) was introduced in [5] for segmenting the semantic scenes with multispectral imagery. The DCNN does not provide the improved performance in the semantic segmentation.

A 3-D wavelet-transform algorithm was introduced in [6] for categorizing the urban multi/hyperspectral images with texture features. The algorithm failed to exhibit a capable scene of the 3-D feature extraction for multi/hyperspectral image analysis.

A Pixon-based image segmentation method was introduced in [7] to segment the multispectral remote sensing data with high accuracy. Though the method minimizes the time complexity, the error rate was not minimized. 
A multi-level fusion of local features extraction and land-use scene classification was presented in [8] with high-resolution images. The method does not increase the classification accuracy for classifying the particular scene in an image. An enhanced kernel principal component analysis (KPCA) technique was introduced in [9] to increase the scene classification accuracy with minimum classification time. The classifications of the event images were not improved.

A stacked de-noising sparse autoencoder (SDSAE) and Fisher vector $(\mathrm{FV})$ was developed in [10] with efficient features for Land-Use Scene Classification. The method does not reduce the error rate in the Land-Use scene classification.

The most significant issues are identified from the above-said literature such as less classification accuracy, high feature extraction time, high error rate, failure to segment the input images and so on. These problems are overcome by introducing a novel machine learning technique called TGKFE-MGBC technique.

The major contribution of the proposed TGKFE-MGBC technique compared to existing methods are summarized as follows,

$>$ The TGKFE-MGBC technique is designed to improve the aerial image scene classification with minimum feature extraction time. This novel contribution is obtained by three different processes. The aerial images with more than two objects are called as multispectral. The object-based segmentation is performed to partition the multispectral images into a number of sub-bands. The Tanimoto similarity coefficient is applied for finding the neighboring pixels similarity to identify the object in sub-band. This process lessens the feature extraction time. The Gaussian Kernelized independent component analysis is applied to project the feature vector from the objects onto the subspace with minimum time.

$>$ To improve aerial image scene classification accuracy, gentleboost machine learning technique is applied. The boosting classifier uses the weak learner as multinomial naïve Bayes probabilistic classifier to categorize the scene into different classes by applying a maximum a posteriori function. The weak learner's results are combined into strong classifier with minimum error rate. This helps to improve aerial image scene classification accuracy and minimizes the false positive rate.

The rest of this paper is arranged into five sections. Section 2 summarizes the related works. Section 3 describes the proposed TGKFE-MGBC technique and the processing way to extract the features for scene classification. First, the whole image is segmented into different subbands. Secondly, the feature learning model is applied to extract the features from the multispectral images. Finally, the machine learning algorithm is applied for scene classification. The simulation settings are presented in section 4 with the aerial image are discussed in Section 5. Finally, the conclusions of the database. The simulation results with different parameters

paper along with the results are presented in section 6 .

\section{RELATED WORKS}

The spectral-structural bag-of-features scene classifier (SSBFC) was developed in [11] with the multispectral aerial images. The classifier failed to consider the spatial relationship models to improve the classification. A superpixel-based multiple local convolution neural network (SML-CNN) method was introduced in [12] for categorizing the multispectral images. The method does not apply any unsupervised deep learning for effective classification.

A new hybrid geometric spatial image representation was introduced in [13] to attain high classification accuracy with the back of features. The method failed to improve the performance since it does not use the geometric spatial features for the large-scale image classification. A Multi-scale completed local binary pattern (MS-CLBP) and a Fisher vector (FV) was introduced in [14] for improving the scene categorization with pattern features. The approach failed to integrate the global and local feature representations for improving the image scene classification.

A kernel feature space representation and support vector machines as well as random forests classifiers were developed in [15] for categorizing the multispectral aerial images. The classification technique does not consider the object-based image analysis for minimizing the feature extraction time. A stratified object-oriented technique was introduced in [16] for categorizing the remote sensing image and feature extraction. Though the technique segments the image objects, the error rate was not minimized.

An object-based convolutional neural network was introduced in [17] for correctly categorizing the high-resolution images with feature learning approach. But the deep feature-based image classifications were not improved. A fast binary coding (FBC) scheme was developed in [18] for efficiently categorizing the remote sensing image scenes with the feature representations. Though the scheme improves the classification accuracy, the feature extraction time was not minimized.

A Deep convolutional neural network (DCNNs) was introduced in [19] for semantic segmentation of the multispectral aerial images. This technique does not use any discriminative frameworks for achieving the high classification performance. In [20], object-based image analysis was designed that concurrently executes segmentation and supervised classification for accurately categorizing the scenes into different classes. But it failed to use other types of segmentation algorithms for accurate scene categorization.

The issues are overcome by introducing a new technique called TGKFE-MGBC. The descriptions of proposed TGKFE-MGBC technique with neat diagram are presented in the next section.

III. TAnimoto GaUssian KERNELIZED FEATURE 


\section{Extraction Based Multinomial GentleBoost TECHNIQUE FOR AERIAL IMAGE SCENE CLASSIFICATION}

Aerial image scene classification is a well-known method in the remote sensing fields. Due to the high resolutions, the geometrical structure of scenes provides a clear vision. An aerial image comprises the earth's surface information with detailed structures of the different objects. Due to the increasing nature of the aerial images with multiple objects, geometrical structures and spatial patterns identification is highly complex in the remote sensing community. Based on this motivation, aerial scene classification is carried out by introducing a novel technique called Tanimoto Gaussian Kernelized Feature Extraction Based Multinomial GentleBoost Classification (TGKFE-MGBC). The proposed TGKFE-MGBC technique uses the aerial images with more than two objects are called as multispectral. The architecture with a different process of the proposed TGKFE-MGBC technique is illustrated in figure 1.

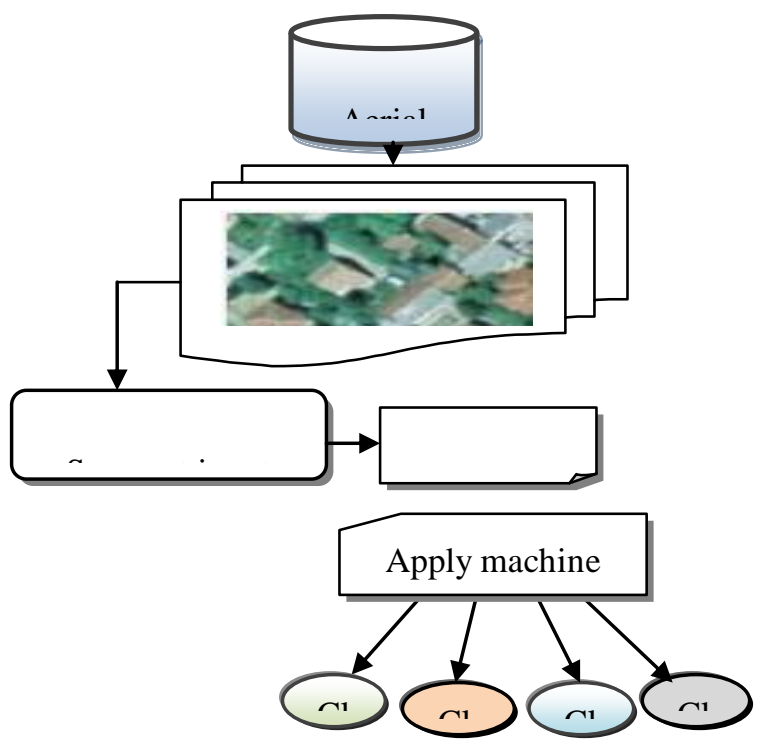

Figure 1 architecture diagram of the aerial image scene classification

Figure 1 depicts the architecture diagram of the proposed TGKFE-MGBC technique to improve the scene classification with high-quality aerial images. The high-quality aerial images $i_{1}, i_{2}, i_{3}, \ldots \ldots i_{n}$ are collected from the image database. Then the input images are segmented into several subbands. After the segmentation, the spatial features like shape, size, color, texture are extracted for further processing with minimum extraction time. Followed by, the numbers of scenes are classified into different classes with the high accuracy from the segmented bands by applying the ensemble classifier. As a result of the classification, the objects are identified on the digital maps. The above-said process of TGKFE-MGBC technique is described in the following sections.

\section{A. Tanimoto pixel similarity object-based segmentation}

The first process in the proposed TGKFE-MGBC technique is to segment the input aerial images. The segmentation is the process to split the entire images into the number of

sub-bands. Each sub-band comprises the different objects. The segmentation process is mainly used to locate objects and their boundaries in high-resolution aerial images. The TGKFE-MGBC technique performs object-based segmentation by measuring the pixels similarity. The objects with similar pixels are segmented in one sub-band. The flow process of the object based segmentation with pixel similarity is shown in figure 2 .

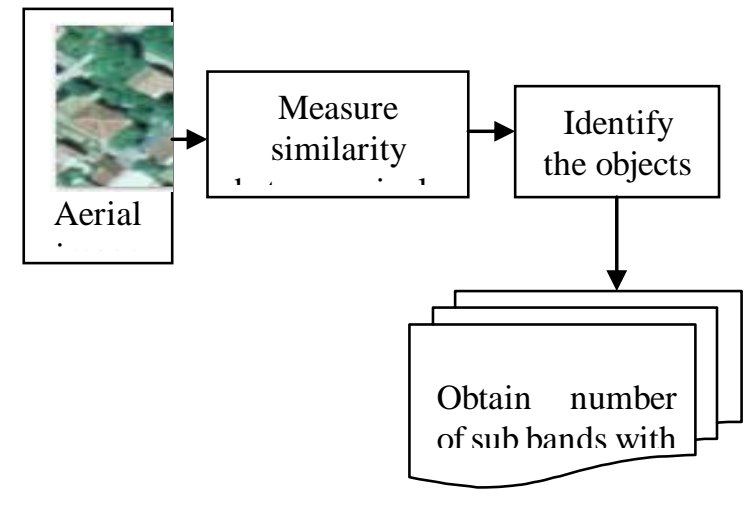

Figure 2 block diagram of aerial image segmentation

Figure 2 illustrates the flow process of aerial image segmentation by measuring the similarity between the two neighboring pixels. The aerial image comprises the number of objects. Each object has a different pixel. Therefore, the object based segmentation is carried out by grouping the similar pixel values. It means that the collection of similar pixels is called an object.

Let us consider the number of images $i_{1}, i_{2}, i_{3}, \ldots, i_{n}$ and each image has number of objects $O_{1}, O_{2}, O_{3}, \ldots, O_{n}$. Each object comprises the number of pixels $p_{l 1}, p_{l 2}, p_{l 3}, \ldots p_{l n}$. The similarity between the two neighboring pixels are computed using Tanimoto similarity is expressed as follows,

$\gamma=\frac{n * \sum p_{l n} p_{l s}}{\sqrt{\sum p_{l 1}{ }^{2}}+\sqrt{\sum p_{l s}{ }^{2}}-\sum p_{l n} p_{l n}}$ (1)

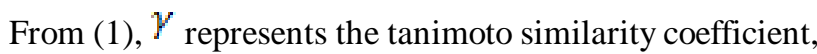
$n$ denotes a number of pixels in images, $p_{l 1}, p_{l 2}$ denotes a two neighboring pixels in an images. $\sum p_{I 1}{ }^{2}$ sum of squared score of the $p_{\pi 1} \sum p_{l 2}{ }^{2}$ denotes the sum of squared score of the $p_{i 2 .} \sum p_{l 1} p_{l 2}$ sum of the product of the paired score of $p_{I 1}$ and $p_{I 2}$. The Tanimoto similarity coefficient provides the output ranges from 0 to +1 , where +1 indicates the high similarity and 0 represents the low similarity. The highest similarity pixels are grouped to identify the object in an image. The segmentations of gray-level objects offer the useful information relates to the surfaces in the scene. By this way, the proposed TGKFE-MGBC technique segments the images into a number of sub-bands with different objects. This helps to minimize the feature extraction time.

Published By: 


\section{B. Gaussian Kernelized Independent Component Analysis based object feature extraction}

After segmenting the aerial images, the features are extracted from the objects in the subbands. In general, the objects have different features like size, shape, texture, color, edges and so on. Feature extraction is the process of transforming the input aerial images into a set of features for classifying the objects with high accuracy. The TGKFE-MGBC technique uses the Gaussian Kernelized independent component analysis (GKICA) for objects feature extraction. GKICA is a computational technique for extracting the object features from the sub-band with minimum time.

$Y=T x$

From (2), ${ }^{Y}$ denotes an m-dimensional feature vector, ${ }^{X}$ is the vector of $n$-dimensional independent components, $T$ represents the weight matrix. The vector of $n$-dimensional independent components is expressed as follows,

$x=\varphi \cdot Y$

From (3), ${ }^{\varphi}$ denotes a mapping function to project the m-dimensional feature vector $Y$ onto the subspace. The Gaussian kernel function is employed as mapping function in a feature space of higher dimensions.

$\varphi=\exp \left(-\beta\left\|Y-Y^{\prime}\right\|^{2}\right)$

$\beta=2 \sigma^{-2}$

From (5), ${ }^{\sigma}$ denotes a variance, $Y$ and $Y^{s}$ represents the feature vectors in some input space, $\left\|Y-Y^{s}\right\|^{2}$ represents the squared Euclidean distance between the two feature vectors. The GKICA model projects the feature vector from the objects onto the subspace. As a result, the features are extracted from the objects for efficient scene classification with minimum complexity.

\section{Gentleboost classification for aerial scene image classification}

The final process of the proposed TGKFE-MGBC technique is to classify the objects as different classes with the extracted features using gentleboost classification. Gentleboost is a machine learning ensemble technique that changes the weak learners into the strong classifier. It is a variant of AdaBoost classifier. A weak learner is a classifier that does not provide the true classification. In contrast, a strong learner provides accurate classification results by combining the weak learners. Therefore, TGKFE-MGBC technique uses the Gentleboost classifier for attaining the strong classification results. The flow process of classification is shown in figure 3 .

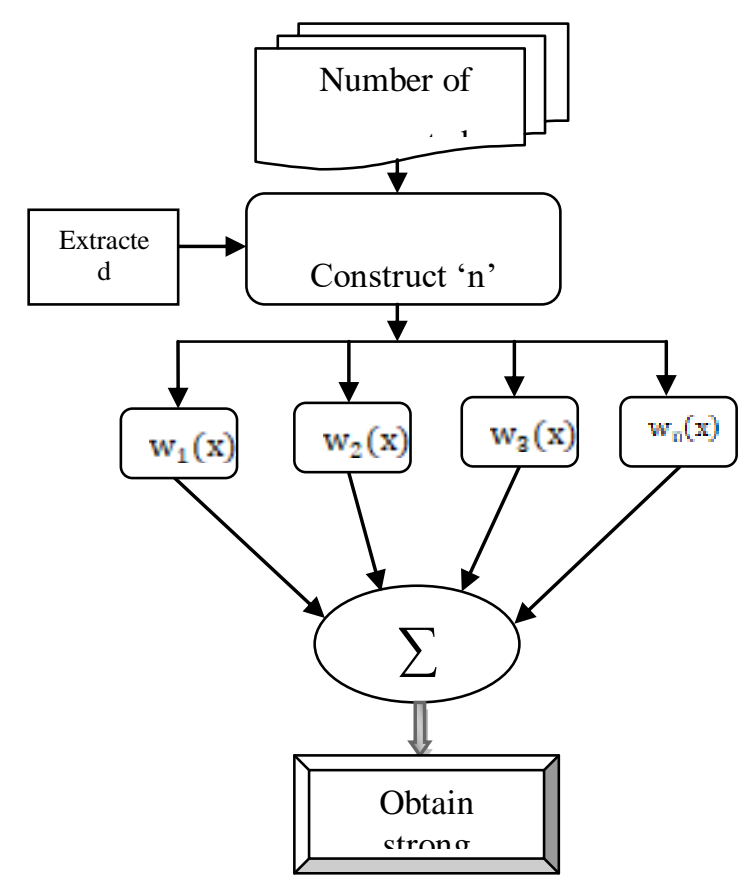

Figure 3 flow process of aerial image scene classification

Figure 3 shows the flow process of aerial image scene classification using ensemble classifier with high classification accuracy with minimum error. Initially, a number of weak learner's i.e. multinomial naïve Bayes probabilistic classifiers are constructed for classifying the scenes. The multinomial naïve Bayes classifier is used for classifying the scene by applying a Bayes' theorem. The numbers of segmented images are considered as input with the extracted features. With a multinomial event approach, scenes are classified into ${ }^{k}$ classes. The multinomial naive Bayes probabilistic classifier performs classification using decision rule to construct the hypothesis. The maximum a posteriori decision rule is used for classifying the images into different classes.

Multinomial naive Bayes classifier comprises the number of training set $\left\{\left(x_{1}, y_{1}\right),\left(x_{2}, y_{2}\right), \ldots,\left(x_{n}, y_{n}\right)\right\}$ where $\mathrm{x}$ denotes the number of segmented images $S I_{1}, S I_{2}, \ldots . S I_{n}$ and ' $\mathrm{y}$ 'denotes a number of possible output multiclass i.e. $\{1,2,3, \ldots . k\}$. By applying the bayes theorem, the conditional probability is expressed as follows,

$p_{r}\left(c l_{j} \mid S I\right)=\sum_{j=1}^{k} \frac{p_{r}\left(S I \mid c l_{j}\right) \varepsilon p_{r}\left(c l_{j}\right)}{p_{r}(S I)}$

From (6), $p_{r}$ denotes a probability, $S I_{i}$ denotes a segmented images, ${ }^{c} l_{j}$ represents the $k$, different classes. $p_{r}\left(S I \mid c l_{j}\right)_{\text {Represents likelihood term and }} p_{r}\left(c l_{j}\right)_{\text {the }}$ prior probability of the classifier, $p_{r}(S I)$ represents the prior probability of the segmented image. By applying the maximum a posteriori 
Bayesian rule, the denominator from equation (6) is normalization term consequently that the posterior Probability Distribution Function integrates to unity. Then, the above equation (6) is modified as follows, $w_{n}(x)=\arg \max \sum_{j=1}^{n} p_{r}\left(c l_{j}\right) p_{r}\left(S I_{i} \mid c l_{j}\right)$ (7)

From (7), ${ }^{w_{n}}(x)$ denotes an output of the weak learners. Then the multinomial probabilistic classifier identifies the maximum probability of the similar images classified into one class while others are classified into different classes. By this way, the multinomial probabilistic classifier correctly classified the images into different classes. As a result, the entire images are classified into a particular class. After the classification, the weak learner has some training error which degrades the classification accuracy and increases the false positive rate. In order to resolve this kind of issues in the scene classification, the gentleboost ensemble classifier combines the outputs of the entire weak learners. The output of the strong classification is expressed as follows,

$y=\sum_{i=1}^{n} w_{i}(x)$

From (8), ${ }^{y}$ denotes a strong classification results, $w_{i}(x)$ represents the output of the weak learner. After combining the entire weak learner output, the similar weight is assigned. For each weak learner, the training error is computed. The error rate is computed based on the squared difference between the actual and estimated output of the each weak learner.

$\beta=\left(a_{0}-w_{i}(x)\right)^{2}$

From (9), $\beta$ denotes a training error of the each weak learner. ${ }^{a}$ represent the actual output of the weak learners and $w_{i}(x)$ represents the estimated output of the weak learner. After computing the error value, the weight of the each weak learner is updated. The weight updating of the weak learner is expressed as follows,

$\tau(t+1)=\tau_{i}(t) \leqslant e^{-y_{i} w_{i}(x)}$

From (10), ${ }^{\tau}(t+1)$ denotes updated weight of the each weak learer, $\tau_{i}(t)$ represents the initial weight of the weak learner. In the weight updating process, the adjustment coefficient is not needed in the gentleboost for the classification process. But the adaboost classifier uses the adjustment coefficient which increases complexity in the classification process. Then the gentleboost classifier finds the weak learner with minimum error. If the weak learner categorizes the scene incorrectly, then the weight is increased. Otherwise the weight of the weak learner decreased. Based on this observation, the accurate classification results are attained as follows,

$y=\arg \min \beta * w_{i}(x)$

From (11), ${ }^{y}$ denotes an output of the strong classifier, $\arg \min \beta$ denotes a minimum error of the weak learner $w_{i}(x)$. The strong classification results show that the segmented images are correctly categorized into the different classes with less error rate. This process improves the aerial image scene classification accuracy and minimizes the false positive rate. The algorithmic procedure of the proposed TGKFE-MGBC technique is described as follows, D

Input: number of aerial images $i_{1}, i_{2}, \dot{i}_{3 y} \ldots i_{n}$ image database

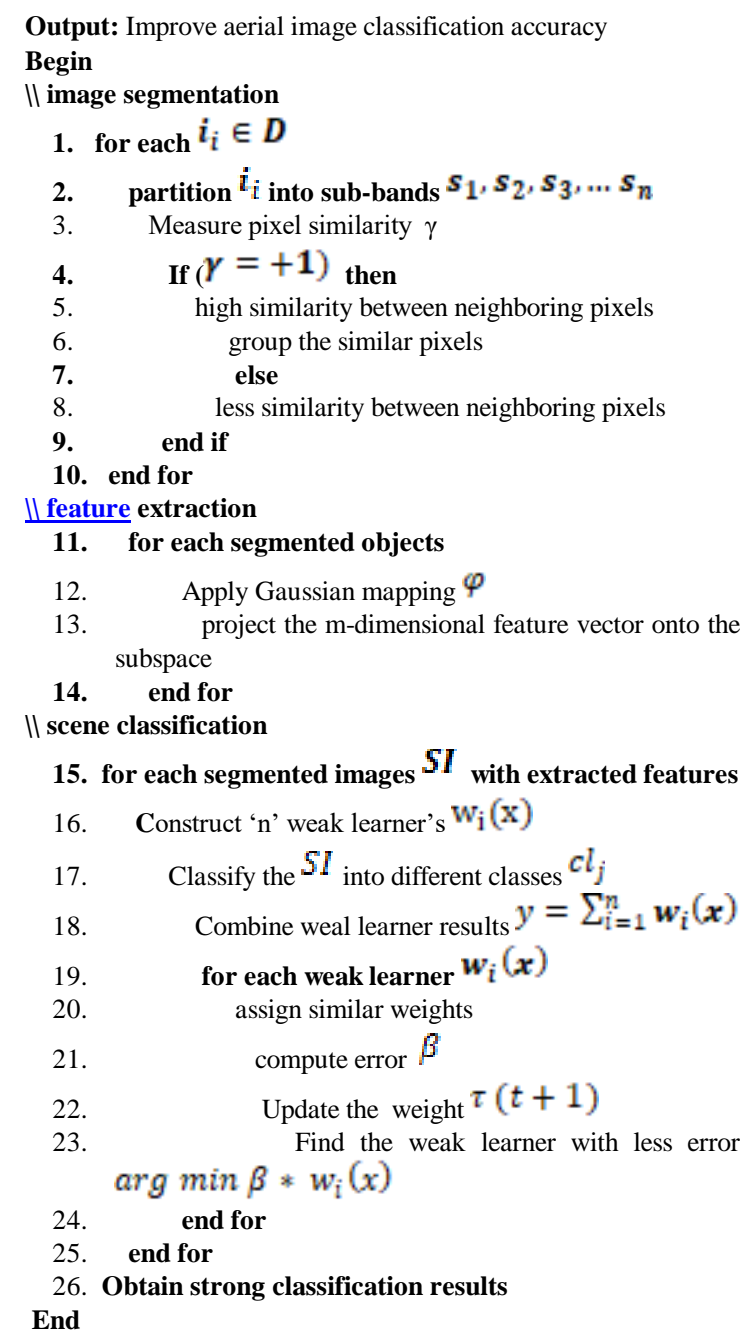

\section{Algorithm 1 Tanimoto Gaussian Kernelized Feature Extraction Based Multinomial GentleBoost scene Classification}

The algorithm 1 describes the process of aerial image scene classification. Before the classification, the whole image is segmented into a number of sub-bands. The object in each subband is identified through the pixel similarity. After segmenting the images, the features are projected into the subspace by applying a Gaussian kernelized mapping function. Finally, the objects in the segmented images are classified by applying the ensemble classifier. At first, the weak learner such as multinomial naïve Bayes probabilistic classifier categorizes the input images into different classes 
through the maximum a posteriori function. After that, the weak learners are summed into the strong classifier. Subsequently, the similar weights are assigned to a weak learner. Then the training error for each weak learner is computed. Followed by, the similar weight is updated for each classifier according to the error value. Based on the weight value, the ensemble classifier finds the weak leaner with less error rate. This helps to obtain strong classification results with the minimum false positive rate.

\section{Simulation SetTings}

The proposed TGKFE-MGBC technique and existing Recurrent Neural Networks (RNN) [1], Block-based semantic classification technique [2] are simulated using MATLAB with RGB aerial images database (http://dsp.etfbl.net/aerial/ ). This aerial image database contains the 606 RGB images with the size of $128 * 128$ pixels. The remote sensed aerial image comprises the various images like buildings, factories, warehouses, fields, trees as well as rivers. There are 200 aerial images are collected from the database for simulation purposes. Totally ten various results are considered with different input images. After collecting the input images, the object based segmentation and feature extraction are performed. Finally, the scenes are classified into different classes such as houses, cemetery, industry, field, river, and trees.

The different simulations results are attained with different parameters such as feature selection time, aerial image scene classification accuracy, and false positive rate with respect to a number of images.

\section{RESULTS AND DISCUSSION}

The results and discussion of TGKFE-MGBC technique and RNN [1], Block-based semantic classification technique [2] is described in this section. The performance results are compared with the various metrics such as feature selection time, aerial image scene classification accuracy, and false positive rate. The different graphical results show that the performance results of proposed and existing methods.

\section{A. Impact of feature extraction time}

Feature extraction time is defined as the amount of time required to extract the features from the input images for accurate scene classification. The feature extraction time is mathematically computed as follows,

\section{feature extraction time $=$ no. of images * $t$ (extracting features from one image)} (12)

From (12), ${ }^{t}$ denotes a time for extracting the features from the one image. The feature extraction time is measured in terms of milliseconds (ms).

\section{B. Sample mathematical calculation for feature extraction time}

> Proposed TGKFE-MGBC technique: No. of images are 25 and the time for extracting the features from one image is $1.2 \mathrm{~ms}$, then feature extraction time is calculated as follows,

\section{feature extraction time $=25 * 1.2 \mathrm{~ms}=30 \mathrm{~ms}$}

Existing RNN: No. of images are 25 and the time for extracting the features from one image is $1.4 \mathrm{~ms}$, then feature extraction time is calculated as follows,

\section{feature extraction time $=25 * 1.4 \mathrm{~ms}=35 \mathrm{~ms}$}

Existing Block-based semantic classification technique: No. of images are 25 and the time for extracting the features from one image is $1.6 \mathrm{~ms}$, then feature extraction time is calculated as follows,

\section{feature extraction time $=25 * 1.6 \mathrm{~ms}=40 \mathrm{~ms}$}

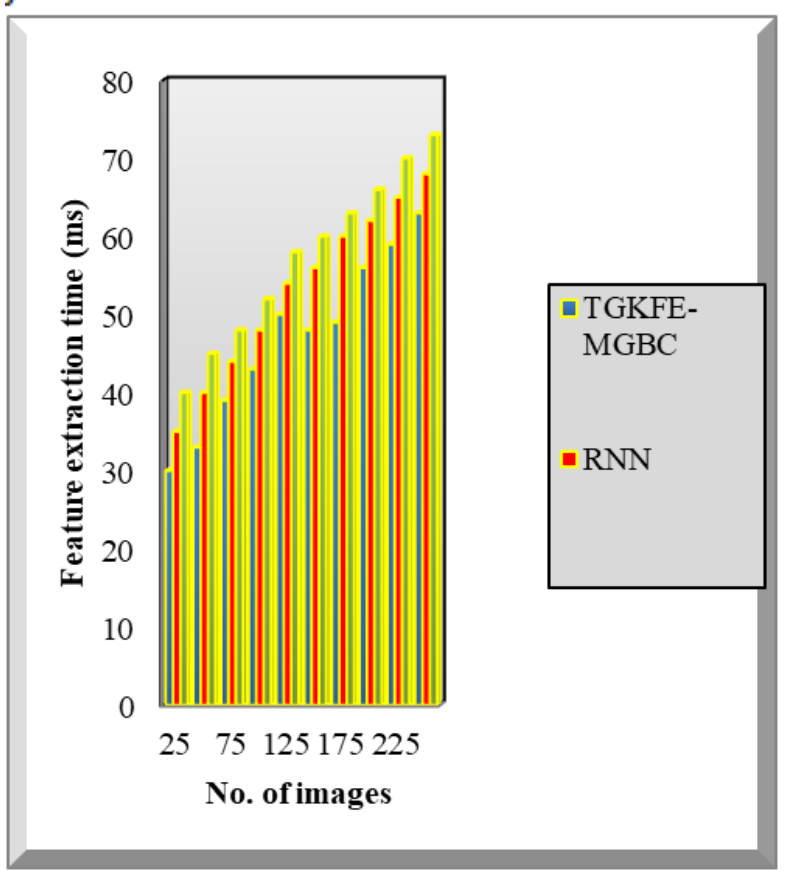

Figure 4 performance results feature extraction time

Figure 4 depicts the performance results feature extraction time versus number of aerial images with three different classification techniques namely TGKFE-MGBC and RNN [1], Block-based semantic classification technique [2]. The numbers of aerial images 25 to 250 are collected from the RGB database. Ten different performance results are attained with various input images. The above graphical results clearly evident that the proposed TGKFE-MGBC technique minimizes the feature extraction time when compared to the existing classification techniques. This significant improvement of the TGKFE-MGBC technique is 
attained by segmenting the input images before the feature extraction. The segmentation is performed by measuring the similarity between the pixels in an image. The similar pixels are grouped for finding the objects in an image. Each spectral band has one object for extracting the features. After that, the Gaussian kernelized independent component analysis is used to project the object features through the mapping function. This process helps to extract the features with minimum time. The comparison results show that the TGKFE-MGBC technique minimizes the feature extraction time by $12 \%$ and $19 \%$ when compared to RNN [1], Block-based semantic classification technique [2] respectively.

\section{5.2 Impact of aerial image scene classification accuracy}

Aerial image scene classification accuracy is defined as the ratio of the number of (i.e. no. of) aerial images are correctly classified to the total number of images. The accuracy is mathematically computed as follows,

$$
\text { AISCA }=\frac{\text { no.of images correctly classified }}{\text { no.of images }} * 100
$$

From (13), AISCA represents aerial image scene classification accuracy. It is measured in terms of percentage (\%).

Sample mathematical calculation for Aerial image scene classification accuracy

> Proposed TGKFE-MGBC technique: No. of images correctly classified is 22 and the total number of the image is 25 , then the aerial image scene classification accuracy is calculated as follows,

$$
\operatorname{AISC} A=\frac{22}{25} \cdot 100=88 \%
$$

Existing RNN: No. of images correctly classified is 21 and the total number of the image is 25 , then the aerial image scene classification accuracy is calculated as follows,

$$
\text { AISC } A=\frac{21}{25} * 100=84 \%
$$

Existing Block-based semantic classification technique: No. of images correctly classified is 20 and the total number of the image is 25 , then the aerial image scene classification accuracy is calculated as follows,

$$
\text { AISCA }=\frac{20}{25} * 100=80 \%
$$

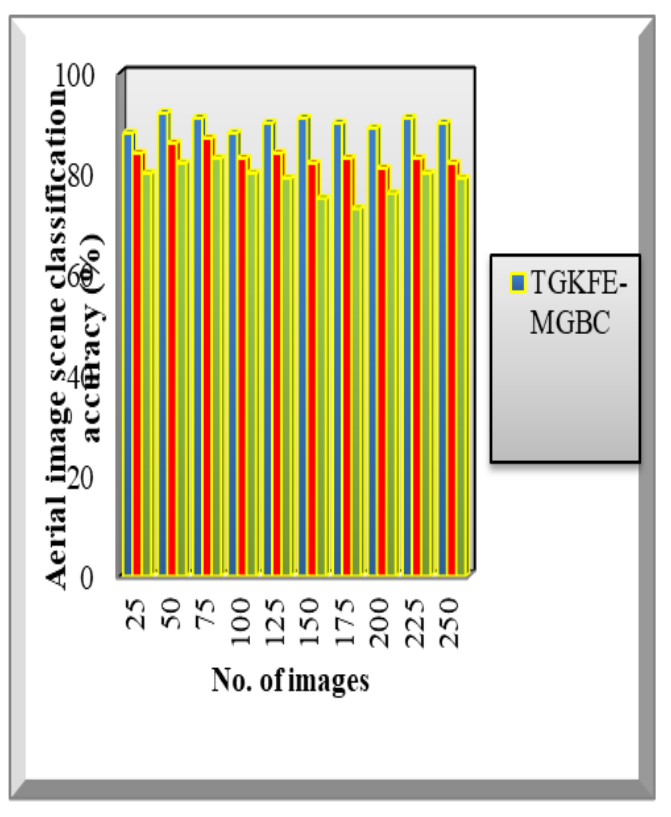

Figure 5 performance results aerial image scene classification accuracy

Figure 5 depicts the performance results of classification accuracy with respect to a number of aerial images taken from the database. The numbers of aerial images are taken as input at ' $x$ ' direction and the corresponding result of classification accuracy is attained at the ' $y$ ' direction. The graphical results show that the TGKFE-MGBC technique increases the performance of aerial image scene classification accuracy. This is because, multinomial gentle boost classification technique is applied to classify the scenes into the different classes such as houses, cemetery, industry, field, river, and trees with the extracted features. Initially, gentleboost classification constructs multinomial naïve Bayes probabilistic classifier finds the maximum likelihood between the classes and the objects. The classifier analyzes the objects in images with their features and categorized into the any one of six classes. The weak learner results are summed into a strong one for improving the classification accuracy. As a result, the objects in a multispectral band image are correctly classified. From the simulation results, the classification accuracy of the proposed TGKFE-MGBC technique is considerably improved by $8 \%$ and $15 \%$ when compared to conventional techniques RNN [1], Block-based semantic classification [2] respectively.

\section{Impact of false positive rate}

The false positive rate is computed as the ratio of a no. of images incorrectly classified to the total no. of images. The false positive rate is computed using the following mathematical equation, 


$$
F P R=\frac{\text { No.of images incorrecly classified }}{\text { No.of images }} 100
$$

From (14), ${ }^{F P R}$ denotes a false positive rate and it is measured in terms of percentage $(\%)$.

Sample mathematical calculation for False Positive Rate

> Proposed TGKFE-MGBC technique: No. of images incorrectly classified is 3 and the total no. of the image is 25 , then the false positive rate is calculated as follows,

$$
F P R=\frac{3}{25} * 100=12 \%
$$

> Existing RNN: No. of images incorrectly classified is 4 and the total no. of the image is 25 , then the false positive rate is calculated as follows,

$$
F P R=\frac{4}{25} \div 100=16 \%
$$

> Existing Block-based semantic classification technique: No. of images incorrectly classified is 5 and the total no. of the image is 25 , then the false positive rate is calculated as follows,

$$
F P R=\frac{5}{25} \div 100=20 \%
$$

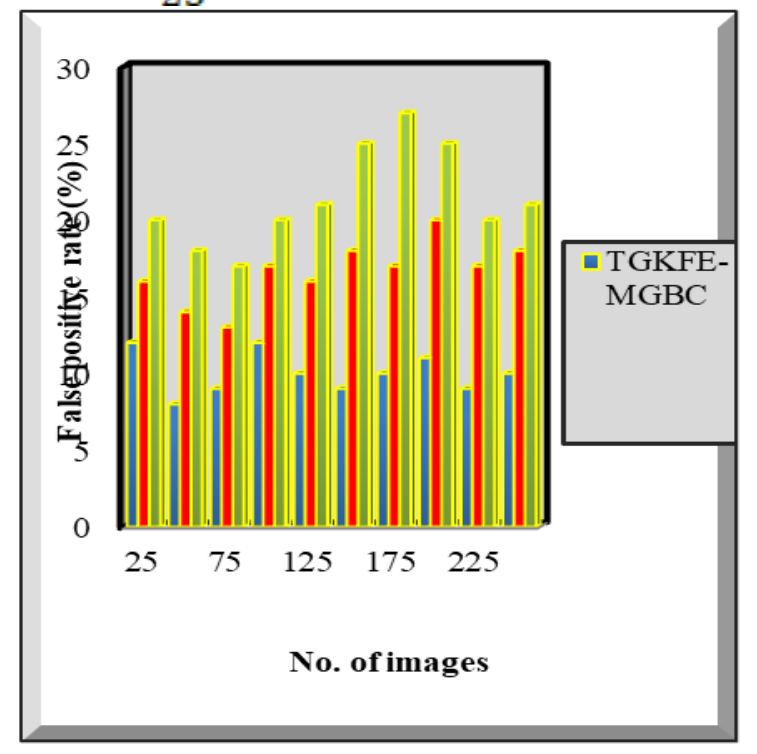

Figure 6 performance results false positive rate

Figure 6 illustrates the simulation results of false positive rate versus a number of aerial images. The above figure illustrates three dissimilar colors shows that the false positive rate of three different methods namely TGKFE-MGBC technique and two conventional classification methods. The graphical results confirm that the proposed TGKFE-MGBC technique minimizes the false positive rate when compared to existing methods. This considerable enhancement is attained by minimizing the error during the scene classification. The boosting classifier combines the entire weak learner results. Then the classifier assigns the similar weights for each weak learner. For each weak learner classification results, the squared difference between the actual and estimated value is computed to find the error. After that, the initial weights of the weak learner are updated according to the error value. Then the strong classifier finds the weak learner with minimum error. This process of the TGKFE-MGBC technique is used to minimize the incorrect scene classification. From the results, the false positive rate gets minimized.

Totally ten various results are carried out to compute the false positive rate. The results of the proposed TGKFE-MGBC technique and conventional methods are compared. The comparison results show that the TGKFE-MGBC technique minimizes the false positive rate by $39 \%$ when compared to RNN [1]. Similarly, the false positive rate of TGKFE-MGBC technique is also reduced by $53 \%$ than the Block-based semantic classification [2] technique.

The above comparison results clearly show that the proposed TGKFE-MGBC technique effectively classifies the aerial images scenes with less feature extraction time and false positive rate.

\section{CONCLUSION}

An efficient machine learning technique called Tanimoto Gaussian Kernelized Feature Extraction Based Multinomial GentleBoost Classification (TGKFE-MGBC) technique is developed for improving the aerial image scene classification accuracy and minimizing the feature extraction time. The input aerial images are segmented into a number of spectral bands with different objects by computing the pixel similarity. The similar pixels are grouped to identify the objects in spectral bands. Then, the features are extracted from the objects using the Gaussian kernel mapping function. Finally, the ensemble technique called multinomial gentleBoost is applied for classifying the scenes in a spectral band with the features. The ensemble classifier categorizes the scenes into different classes by constructing the weak learners with high classification accuracy and minimum training error. Simulation results are carried out with RGB aerial image database with different parameters such as feature extraction time, aerial image scene classification accuracy and false positive rate. The simulation result reveals that the TGKFE-MGBC technique significantly increases the aerial image scene classification accuracy and minimizes the feature extraction time as well as false positive rate when compared to state-of-art methods. 


\section{REFERENCES}

1. Emmanuel Maggiori, Guillaume Charpiat, Yuliya Tarabalka, and Pierre Alliez, "Recurrent Neural Networks to Correct Satellite Image Classification Maps", IEEE Transactions on Geoscience and Remote Sensing, Volume 55, Issue 10, October 2017, Pages 5913 - 5924

2. Aleksej Avramovic and Vladimir Risojevic, "Block-based semantic classification of high-resolution multispectral aerial images", Signal, Image and Video Processing, Springer, Volume 10, Issue 1, January 2016, Pages 75-84

3. Yunlong Yu and Fuxian Liu, "A Two-Stream Deep Fusion Framework for High-Resolution Aerial Scene Classification", Computational Intelligence and Neuroscience, Hindawi, Volume 2018, January 2018 , Pages 1-13

4. Jinyi Zou,Wei Li,Chen Chen,Qian Du, "Scene classification using local and global features with collaborative representation fusion", Information Sciences, Volume 348, 2016, Pages 209-226

5. Ronald Kemker, Carl Salvaggio, Christopher Kanan, "Algorithms for semantic segmentation of multispectral remote sensing imagery using deep learning", ISPRS Journal of Photogrammetry and Remote Sensing, Elsevier, Volume 145, 2018, Pages 60-77

6. Xian Guo , Xin Huang, Liangpei Zhang, "Three-Dimensional Wavelet Texture Feature Extraction and Classification for Multi/Hyperspectral Imagery", IEEE Geoscience and Remote Sensing Letters, Volume 11 , Issue 12, 2014, Pages 2183 - 2187

7. Amin Zehtabian and Hassan Ghassemian, "An Adaptive Pixon Extraction Technique for Multispectral/Hyperspectral Image Classification", IEEE Geoscience and Remote Sensing Letters , Volume 12 , Issue 4, 2015 , Pages $831-835$

8. Chen Lu, Xiaomei Yang, Zhihua Wang, Zhi Li, "Using multi-level fusion of local features for land-use scene classification with high spatial resolution images in urban coastal zones", International Journal of Applied Earth Observation and Geo-information, Volume 70, 2018, Pages 1-12

9. Baoyu Dong and Guang Ren, "A New Scene Classification Method Based on Local Gabor Features", Mathematical Problems in Engineering, Hindawi Publishing Corporation, Volume 2015, April 2015, Pages 1-14

10. Hang Wu, Baozhen Liu, Weihua Su, Wenchang Zhang, Jinggong Sun, "Deep Filter Banks for Land-Use Scene Classification", IEEE Geoscience and Remote Sensing Letters, Volume 13 , Issue 12 , 2016, Pages 1895 1899

11. Bei Zhao, Yanfei Zhong, Liangpei Zhang, "A spectral-structural bag-of-features scene classifier for very high spatial resolution remote sensing imagery", ISPRS Journal of Photogrammetry and Remote Sensing, Elsevier, Volume 116, 2016, Pages 73-85

12. Wei Zhao, Licheng Jiao, Wenping Ma, Jiaqi Zhao, Jin Zhao, Hongying Liu, "Superpixel-Based Multiple Local CNN for Panchromatic and Multispectral Image Classification", IEEE Transactions on Geoscience and Remote Sensing, Volume 55 , Issue 7 , 2017, Pages 4141 - 4156

13. Nouman Ali, Bushra Zafar, Faisal Riaz, Saadat Hanif Dar, Naeem Iqbal Ratyal, Khalid Bashir Bajwa, Muhammad Kashif Iqbal, Muhammad Sajid, "A Hybrid Geometric Spatial Image Representation for scene classification", PLoS ONE, Volume 13, Issue 9, 2018, Pages 1-27

14. Longhui Huang, Chen Chen, Wei Li and Qian Du, "Remote Sensing Image Scene Classification Using Multi-Scale Completed Local Binary Patterns and Fisher Vectors", Remote Sensing, Volume 8, Issue 6,2016, Pages 1-17

15. Sergio Bernabé, Prashanth Reddy Marpu , Antonio Plaza, Mauro Dalla Mura , Jon Atli Benediktsson, "Spectral-Spatial Classification of Multispectral Images Using Kernel Feature Space Representation”, IEEE Geoscience and Remote Sensing Letters, Volume 11 , Issue 1 ,2014, Pages $288-292$

16. Wen Zhou, Dongping Ming, Lu Xu, Hanqing Bao, and Min Wan, "Stratified Object-Oriented Image Classification Based on Remote Sensing Image Scene Division", Journal of Spectroscopy, Hindawi, Volume 2018, June 2018, Pages 1-11

17. Wenzhi Zhao, Shihong Du, and William J. Emery, "Object-Based Convolutional Neural Network for High-Resolution Imagery Classification", IEEE Journal of Selected Topics in Applied Earth Observations and Remote Sensing, Volume 10 , Issue 7, 2017, Pages $3386-3396$

18. Fan Hu , Gui-Song Xia , Jingwen Hu, Yanfei Zhong and Kan Xu , "Fast Binary Coding for the Scene Classification of High-Resolution Remote Sensing Imagery”, Remote Sensing, Volume 8, Issue 7, 2016, Pages 1-24

19. Ronald Kemker, Carl Salvaggio, Christopher Kanan, "Algorithms for semantic segmentation of multispectral remote sensing imagery using deep learning", ISPRS Journal of Photogrammetry and Remote Sensing, Elsevier, Volume 145, Part A, 2018, Pages 60-77

20. Daniel Capella Zanotta, Maciel Zortea, Matheus Pinheiro, Ferreira, "A supervised approach for simultaneous segmentation and classification of remote sensing images", ISPRS Journal of Photogrammetry and Remote Sensing, Elsevier, Volume 142, 2018, Pages 162-173

\section{AUTHORS PROFILE}

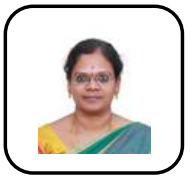

Mrs.A.Gokila Vani is a Assistant Professor in Department of Computer Science at KG College of Arts and Science, Coimbatore. She did her MCA degree at Bharathiar University. She started her teaching profession at KG College of Arts and Science, Coimbatore in 2008. Her teaching areas are Visual Programming, Software Engineering and Software Project Management, Data Mining, C Programming and Java Programming. She did her M.Phil. at Bharathiar University, Coimbatore. She is currently pursuing $\mathrm{Ph} . \mathrm{D}$. at Bharathiar University under the guidance of Dr.V.Saravanan. She has over 4 publications in international referred journals. She has presented papers at various conferences and published book.

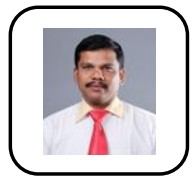

Dr.V. Saravanan is a Professor and Head on Department of Information Technology at Hindusthan College of Arts and Science, Coimbatore. He did his M.Sc in computer science at the Bharadhidasan University, Trichy. And he did his MCA at the Periyar University, Salem. He started his teaching profession at Thanthai Hans Roever College Perambalur, Trichy in 1999. Later in 2004 he joined in Hindusthan College of Arts and Science, Coimbatore. His teaching areas are Networking and mobile computing as it is his main area of interest. He did his M.Phil and Ph.D in at ManonmaniamSundaranar university, Tirunelveli. His Ph.D was on Wireless Networking in video streaming. He has over 36 publications in internationals referred journals. He has presented research papers at conferences, published articles and papers in various journals. $\mathrm{He}$ is renowned key note address in both national and international conferences 